\title{
Cellular phenotyping of chronic rhinosinusitis with nasal polyps*
}

\author{
Hongfei Lou' ${ }^{1,2}$, Yifan Meng ${ }^{1,2 \#}$, Yingshi Piao 3 , Nan Zhang 4 , Claus Bachert4 \\ Chengshuo Wang ${ }^{1,2}$, Luo Zhang ${ }^{1,2,5}$ \\ Rhinology 54: 150-159, 2016 \\ DOI:10.4193/Rhino15.271 \\ ' Department of Otolaryngology Head and Neck Surgery, Beijing TongRen Hospital, Capital Medical University, Beijing 100730, PR \\ China \\ *Received for publication: \\ September 1, 2015 \\ 2 Beijing Key Laboratory of nasal diseases, Beijing Institute of Otolaryngology, Beijing 100005, PR China \\ Accepted: October 12, 2015 \\ ${ }^{3}$ Department of Pathology, Beijing TongRen Hospital, Capital Medical University, Beijing 100730, PR China \\ ${ }^{4}$ Upper Airways Research Laboratory, Department of Oto-Rhino-Laryngology, Ghent University Hospital, De Pintelaan 185, 9000 \\ Ghent, Belgium \\ \# These authors contributed \\ ${ }^{5}$ Department of Allergy, Beijing TongRen Hospital, Capital Medical University, Beijing 100730, PR China \\ equally to the study
}

\begin{abstract}
Background: Defining the phenotypes of chronic rhinosinusitis with nasal polyps (CRSWNP) with prognosis may lead to delivery of personalized treatment. This study aimed to identify cellular phenotypes of CRSwNP using cluster analysis and define an algorithm for different clusters associated with polyp recurrence.
\end{abstract}

Methods: Overall, 366 patients with CRSwNP were enrolled in this retrospective analysis. Eighteen variables, including clinical characteristics and tissue/peripheral inflammatory cells assessments, were selected for factor analysis. Unsupervised cluster analysis was performed after variables reduction and standardization and differences in polyp recurrence during follow-up for a minimum of 24 months were analysed among clusters. Discriminant analysis was further used to develop a clinically useful algorithm for predicting clustering.

Results: Five phenotypic clusters were identified. Clusters 1 and 2 were plasma cell-dominant and lymphocyte-dominant phenotypes, respectively. Cluster 3 revealed a mixed inflammatory pattern. Cluster 4 was characterized by infiltration of predominantly neutrophils. Cluster 5 was characterized by a marked tissue eosinophilia and highest recurrence rate of $98.5 \%$. The clinical algorithm predicted clustering with $93.7 \%$ accuracy.

Conclusions: Chinese CRSwNP patients may be classified into five phenotypes with different polyp recurrence rates, based on the presence of predominantly plasma cells, lymphocytes, neutrophils, eosinophils or mixed inflammatory cells in polyps.

Key words: chronic rhinosinusitis with nasal polyps (CRSwNP), clinical algorithm, cluster analysis, inflammatory cell types, polyp recurrence

\section{Introduction}

Chronic rhinosinusitiswith nasal polyps (CRSwNP) is a common and complex disease with heterogeneous sinonasal inflammation ${ }^{(1,2)}$. The inflammatory patterns of nasal polyps are roughly designated to be eosinophilic and neutrophilic based on the predominant inflammatory cell type, and mediator or cytokine expression ${ }^{(3,4)}$. Indeed, cellular (eosinophils) and molecular (leukotriene C4) markers, in conjunction with objective and 
subjective clinical parameters, have been previously analyzed to establish the complicated classification system for chronic rhinosinusitis $(\mathrm{CRS})^{(5)}$. Wormald and colleagues ${ }^{(6)}$ recommended that the extent of mucosal inflammatory load, especially tissue eosinophils, should be considered as the most important indicator for functional or radical surgical approach. Furthermore, the inflammatory phenotype has great significance in determining steroid responsiveness and surgical outcome ${ }^{(3,7-9)}$. However, the numbers of eosinophils, neutrophils and other inflammatory cells, vary greatly in nasal polyps, and accurate algorithms of CRSwNP classification are not clear-cut. Thus, a better insight into different inflammatory patterns might allow the precise identification of subgroups in terms of their response to current medical and surgical interventions; which could possibly lead to individualization of therapy with the potential for more effective treatment and better patient outcomes.

As a multifactorial disorder, CRSwNP may be classified clinically with less bias using cluster analysis, an unsupervised statistical approach in which individual subjects are grouped based on multiple similarities in clinical or biological measures. Although studies by Nakayama and colleagues ${ }^{(10)}$ and Soler and colleagues ${ }^{(11)}$ have classified CRS patients into distinct phenotypes using cluster analysis, these studies didn't attempt to classify CRS prognosis. Thus, to elucidate CRSwNP inflammatory spectrum with corresponding prognosis, we performed a cluster analysis including long-term follow-up outcomes.

Firstly, our study aimed to identify subgroups of CRSwNP subjects who share not only phenotypic traits but also similar inflammatory cell patterns using an unsupervised cluster approach. Secondly, the study aimed to define a simple algorithm to classify patients into identified clusters correlated with polyp recurrence.

\section{Materials and methods}

\section{Study cohort}

The medical records of 366 patients ( 258 men and 106 women), aged 18-78 years old ( $45.9 \pm 12.5$ years old) with CRSwNP, who had undergone endoscopic sinus surgery (ESS) at Beijing TongRen Hospital, from January 2011 to January 2013, were reviewed retrospectively for preoperative demographic characteristics and medical history. Overall, 71 patients (19.4\%) had concomitant asthma and none were treated with antibiotics or corticosteroids within a 4-week period before surgery. Records of patients who had unilateral disease, allergic fungal rhinosinusitis, antrochoanal polyps, or cysts were excluded from the study. Prior to surgery, nasal symptoms (including nasal obstruction, nasal discharge, olfactory dysfunction and headache/facial pain) were assessed using a visual analogue scale (VAS) of 0 to 10 and the size of nasal polyps was graded by an independent observer using the Davos score ${ }^{(12)}$. Blood samples were also taken prior to surgery and a complete peripheral blood cell count and a differential cell count was performed using an automated analyser. Preoperative computed tomography (CT) scans (Brilliance 64-section CT system, Philips Healthcare, Best, The Netherlands) were scored according to Lund-Mackay staging system ${ }^{(13)}$. Atopy was confirmed based on a positive skin prick test to a panel of common allergens (Allergopharma, Reinbeck, Germany) and/ or positive serum antigen-specific IgE, measured by ImmunoCAP 100 system (Pharmacia, Uppsala, Sweden). Oral fractional exhaled nitric oxide (FENO) was measured using the NIOX electrochemical analyser (Niox; Aerocrine, Solna, Sweden) at a flow rate of $50 \mathrm{ml} / \mathrm{s}$. ESS was performed by the same surgeon on all patients based on the findings by preoperative $\mathrm{CT}$ and recurrence of polyps was characterised one year after surgery by nasal endoscopy as the presence of diseased mucosa (nasal polyps, mucopurulent secretions, and/or inflamed mucosa), together with bothersome symptoms persisting for at least one month and the need for maximal medical treatment ${ }^{(9)}$. All patients were followed-up postoperative for a minimum of 24 months (35.03 \pm 5.14 months).

The study was approved by the Medical Ethics Committee of Beijing TongRen Hospital and all patients had provided written informed consent prior to collection of their data.

\section{Histological evaluation of polyp tissue}

Samples of polyp tissues were processed for histological evaluation by $\mathrm{H} \& \mathrm{E}$ stain and all stained samples were observed by two independent pathologists, who were blinded to the clinical diagnosis and characteristics of the patients. Total cell counts were recorded as the mean of the counts for 10 non-overlapping fields in the lamina propria and infiltrating inflammatory cells; including eosinophils, neutrophils, plasma cells and lymphocytes; were assessed by bright-field light microscopy (BX51, Olympus, Japan) at $\times 400$ magnification. The numbers of inflammatory cell types were calculated as the percentage of total inflammatory cells present.

\section{Statistical analysis}

Factor analysis was performed on 18 variables; including demographic data (age, gender), medical comorbidities and related parameters (asthma, atopy, FENO), subjective and objective CRSwNP severity metrics (symptoms, CT, endoscopy), and tissue/ peripheral inflammatory cells counts; to allow reduction in the number of variables. The first three factors (eigenvalues were $3.31,1.21$ and 0.84 , respectively) providing an accumulative contribution of $87 \%$ were selected. Orthogonal varimax rotation was performed, resulting in four variables, i.e. tissue eosinophil percentage (Eos\%), tissue neutrophil percentage (Neu\%), tissue plasma cell percentage (Pla\%) and tissue lymphocyte percentage (Lym\%), whose factor loadings were greater than 0.4 in the selected factors. Normality standardization was performed by computing $Z$ score for each variable, such that all variables were 


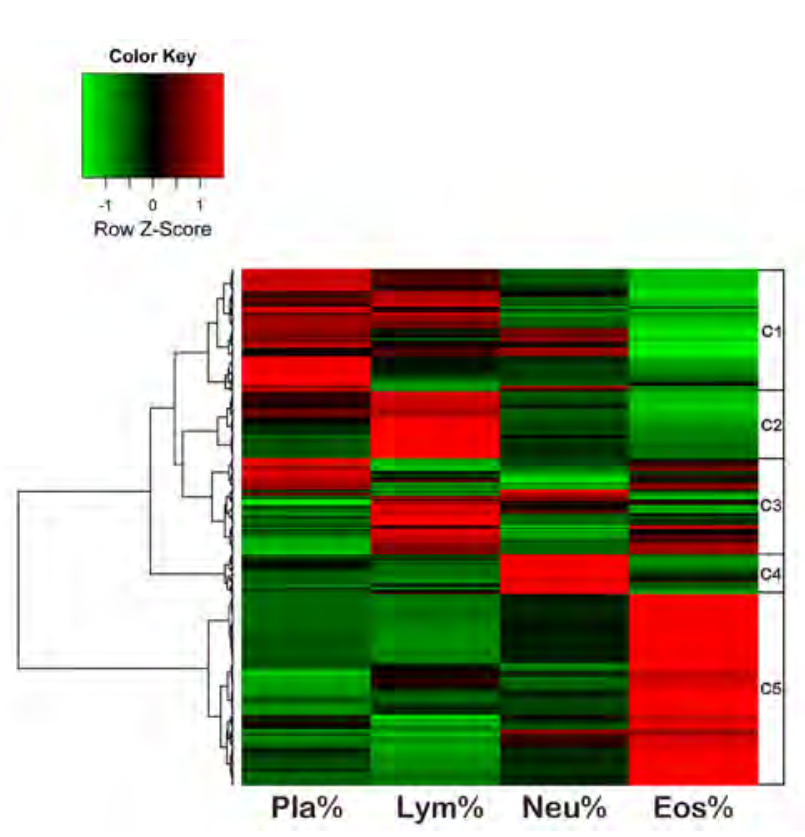

Figure 1. Hierarchical clustering of four mucosal inflammatory cells. Shown is a heat map of clustering mucosal inflammatory cell percentages in nasal polyps. Each row represents one subject, and the individual inflammatory cell proportions are shown in each column, with green being low and red being high. Left, Dendogram showing similarity of groups. Right, Five clusters (C) are indicated by vertical bars (C1-C5). Eos\%, eosinophil percentage. Neu\%, neutrophil percentage. Pla\%, plasma cell percentage. Lym\%, lymphocyte percentage.

of similar dimension in further analysis.

Hierarchical clustering method with Euclidean similarity measure and Ward minimum-variance linkage was performed using Stata 12 (StataCorp LP, College Station, TX, USA). The optimal number of clusters was determined by the maximum Calinski/ Harabasz's pseudo-F index andthe result of cluster analysis was rendered as a heat map, which was drawn by using R3.1.2 (The R Foundation for Statistical Computing, http://www.r-project. org/). After defining the clusters, polyp recurrence was analysed in the different clusters.

Fisher's linear discrimination analysis with group-size-proportional prior probabilities was used to investigate whether the parameters significantly discriminated among the clusters. We used an F-value entry probability of 0.05 and removal probability of 0.10 according to the Fisher's method. Multivariate classification tree analysis was performed on four variables to assess the classification performance of CRSWNP.

The variables between the clusters were compared by one-way analysis of variance, Tukey's test and Chi-square test using SPSS version 19.0 (IBM Corp., Armonk, NY, USA). Data were expressed as median and interquartile range, unless otherwise specified. Statistical significance was set at a $P$ value $\leq 0.05$.
Table 1. Calinski and Harabasz's pseudo-F index.

\begin{tabular}{|cc|}
\hline $\begin{array}{c}\text { Number of Clusters } \\
2\end{array}$ & $\begin{array}{c}\text { Calinski and Harabasz's } \\
\text { Pseudo-F }\end{array}$ \\
\hline 3 & 237.66 \\
\hline 4 & 266.73 \\
\hline 5 & 275.51 \\
\hline 6 & 295.77 \\
\hline 7 & 289.59 \\
\hline 8 & 267.36 \\
\hline 9 & 268.84 \\
\hline 10 & 264.90 \\
11 & 260.50 \\
\hline 12 & 249.94 \\
\hline 13 & 240.07 \\
14 & 245.86 \\
\hline 15 & 241.95 \\
\hline & 253.90 \\
\hline
\end{tabular}

\section{Results}

Inflammatory cell patterns in $\mathbf{5}$ CRSwNP clusters

Calinski and Harabasz pseudo-F index was calculated using the agglomerative cluster approach, and showed that 4 to 6 clusters had the top three highest index values, with 5 clusters being the highest index (Table 1). Unsupervised agglomerative hierarchical clustering, which groups each subject on the basis of mathematical similarities of mucosal inflammatory cell proportion to the others, indicated that five clusters displaying different inflammation patterns were present as follows: cluster 1 with a plasma cell-dominant phenotype, cluster 2 with a lymphocytedominant phenotype, cluster 3 with a mixed inflammation phenotype, cluster 4 with a neutrophil-dominant phenotype, and cluster 5 with an eosinophil-dominant phenotype (Figure 1). Figure 2 shows typical pathologic images for the respective clusters.

\section{Clinical characteristics of 5 clusters}

To determine whether the patients within these subgroups were representative of distinct phenotypes of CRSWNP, the clinical and demographic features of these five clusters were compared (Table 2). The 5 clusters were not significantly different with regard to age distribution, Lund-Kennedy endoscopic score, atopic status, peripheral lymphocytes counts and peripheral plasma cells counts across the clusters. However, cluster 3 and cluster 5 comprised significantly high proportion of tissue eosinophils, compared to the other three clusters (Figure 3A). Peripheral eosinophil proportion, oral FENO, and olfactory scores also mirrored similar patterns in these clusters (Figure 3B, C, D). Overall, cluster 1 contained $23.8 \%(n=87)$ of patients; with $67.8 \%(59 / 87)$ being male. Plasma cells percentage was highest of the 5 clusters and the proportions of tissue lymphocytes and 

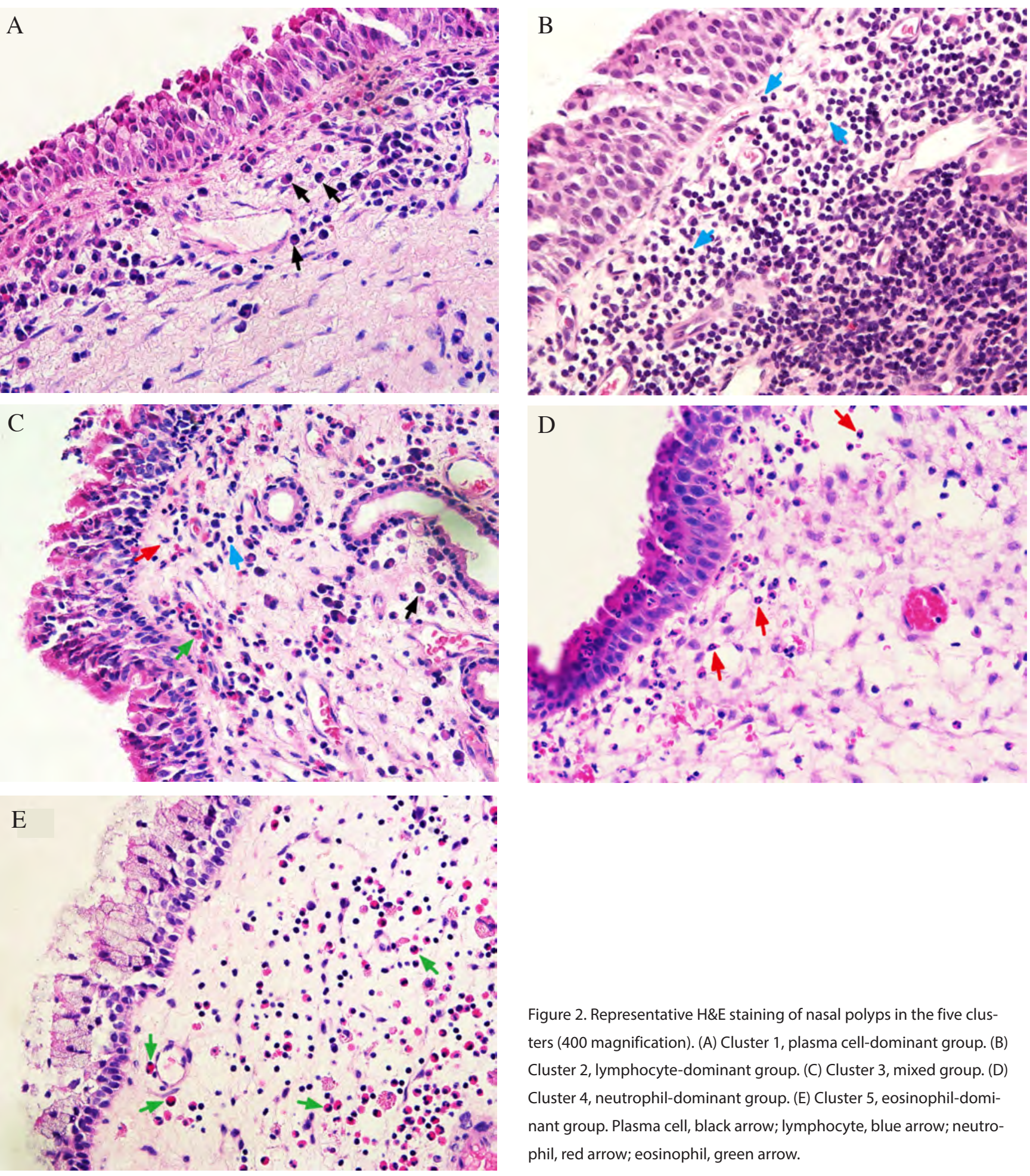

Figure 2. Representative H\&E staining of nasal polyps in the five clusters (400 magnification). (A) Cluster 1, plasma cell-dominant group. (B) Cluster 2, lymphocyte-dominant group. (C) Cluster 3, mixed group. (D) Cluster 4, neutrophil-dominant group. (E) Cluster 5, eosinophil-dominant group. Plasma cell, black arrow; lymphocyte, blue arrow; neutrophil, red arrow; eosinophil, green arrow.

eosinophils were low. The symptom and CT scores of patients in this cluster were low, as were the co-morbid asthma incidence (4.6\%) and oral FENO concentration. Six patients (6.9\%) experienced polyp recurrence in cluster 1 .

Cluster 2 contained $12.8 \%(n=47)$ of patients, of whom $83 \%$ were male. This cluster was characterized by lymphocyte-dominant inflammation, with low values for peripheral granulocytes counts (eosinophils and neutrophils), preoperative CT scores and symptom scores. Tissue eosinophils count was similar to cluster 1, whereas tissue neutrophils proportion was significantly lower. Scores of nasal obstruction, rhinorrhea, and headache were comparable to cluster 1 , but significantly less than for cluster 4 and cluster $5(P<0.01)$. The incidence of co-morbid asthma (2.1\%) was the least in five clusters. Cluster 2 contained 3 patients (6.4\%) with polyp recurrence.

Cluster 3 comprised $18.6 \%(n=68)$ of all patients, of whom 

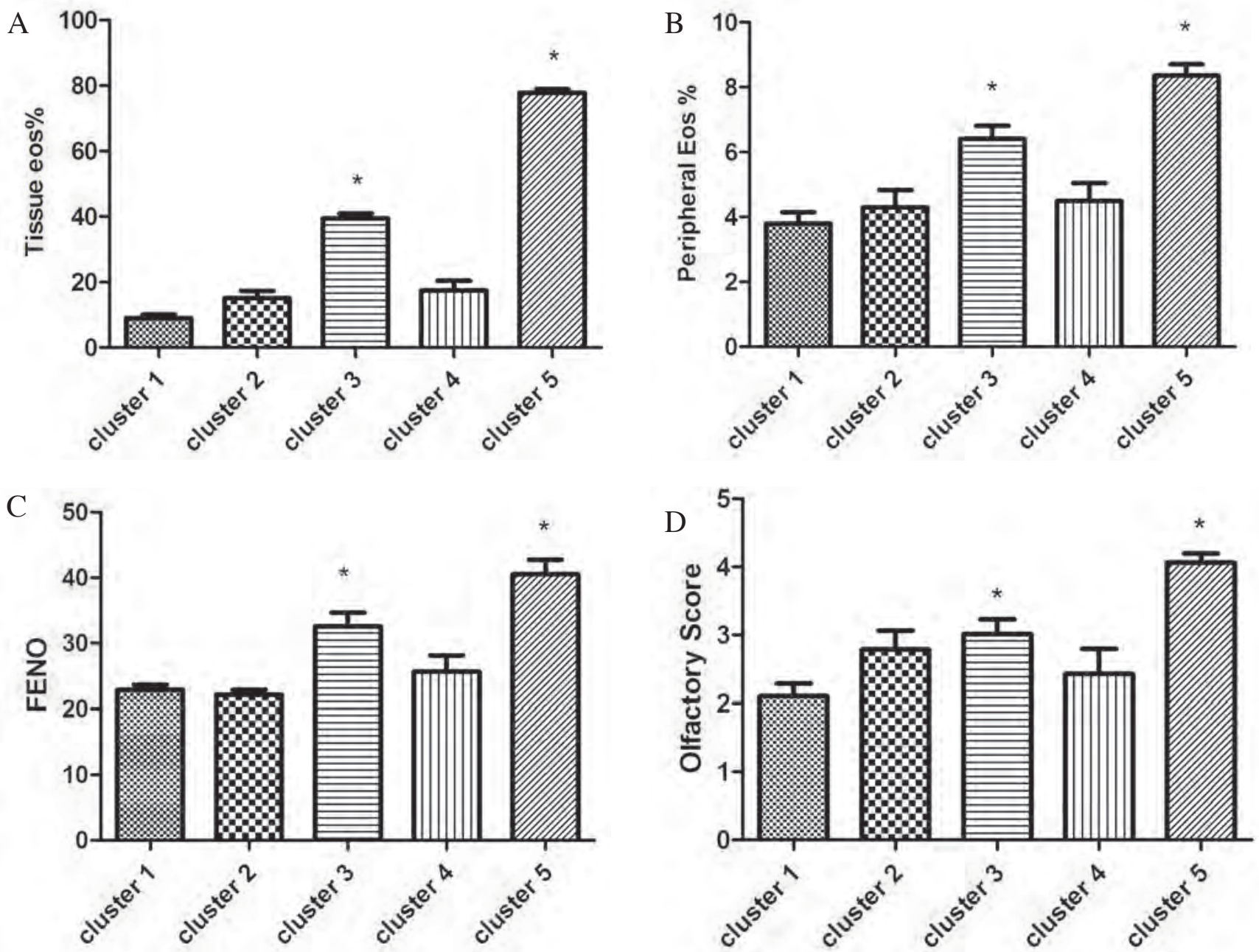

Figure 3. Tissue Eos\%, peripheral Eos\%, FENO, and olfactory score comparison shared the same pattern in different clusters. (A) Tissue Eos\%. (B) Peripheral Eos\%. (C) FENO. (D) Olfactory score.

$86.8 \%$ were male. This cluster showed mixed inflammatory pattern in nasal polyps and apart from cluster 5 , had higher levels of mucosal eosinophil count, peripheral eosinophil count, oral FENO and olfactory score than clusters 1, 2 and 4. Co-morbid asthma was more common (19.1\%), compared to cluster 1 and 2 and $75 \%$ cases $(51 / 68)$ reported polyp recurrence during followup in this cluster.

Cluster 4 comprised only $7.7 \%(n=28)$ of the total patients and was the smallest group characterized by a predominant infiltration of neutrophils. Subjectively, rhinorrhea, nasal obstruction and headache/facial pain scores were elevated, whereas olfactory dysfunction was mild for clusters 1 and 2. Despite lower airway inflammation, co-morbid asthma was present in $17.9 \%$ of the patients in this group and the rate of polyp recurrence was $46.4 \%(13 / 28)$.

Cluster 5 comprising $37.2 \%(n=136)$ of all patients was the largest cluster and had the most women (36.0\%). Tissue eosinophil count and peripheral eosinophil count displayed the highest values. The worst subjective symptoms and objective CT scores in this cluster indicated more severe disease compared to all other clusters. The incidence of co-morbid asthma was most frequent in this cluster (34.6\%), and FENO concentration the highest of all clusters. During the last two years, 134 out of 136 patients (98.5\%) experienced polyp recurrence.

\section{Discriminant analysis}

Discriminant analysis identified the proportion of eosinophils, neutrophils, plasma cells and lymphocytes in polyp tissue as the four strongest predictors of clustering (Table 3). The accuracy of the discriminant function models for these four variables was 94\% for original models and $93.7 \%$ for cross-validated models (Table 4). Binary classification tree analysis was performed using the four variables to develop a clinical algorithm for grouping patients into appropriate clusters. The resulting algorithm (Figure 4), with cut-off values in each split, accurately predicted the assignment of $93.7 \%$ (343/366) patients to their appropriate clusters (Table 5). 
Table 2. Demographics and clinical characteristics of CRS subgroups by cluster analysis.

\begin{tabular}{|c|c|c|c|c|c|c|}
\hline & Cluster 1 & Cluster 2 & Cluster 3 & Cluster 4 & Cluster 5 & $P$ value \\
\hline No. of subjects & 87 & 47 & 68 & 28 & 136 & \\
\hline Age (yrs)凹 & $46.36 \pm 15.23$ & $47.53 \pm 12.28$ & $45.91 \pm 13.46$ & $41.36 \pm 13.39$ & $45.84 \pm 11.86$ & 0.389 \\
\hline \multirow[t]{2}{*}{ Gender(M/F) } & $59 / 28$ & $39 / 8$ & $59 / 9$ & $22 / 6$ & $87 / 49$ & $0.003^{*}$ \\
\hline & $(32.2 \%)$ & (17.0\%) & (13.2\%) & (21.4\%) & (36.0\%) & 0.346 \\
\hline Atopy $(\mathrm{Y} / \mathrm{N})$ & $11 / 76$ & $11 / 36$ & $9 / 59$ & $3 / 25$ & $26 / 110$ & 0.743 \\
\hline Lund-Kennedy score & $5(4-5)$ & $5(4-5)$ & $5(4-5)$ & $5(4-6)$ & $5(4-5)$ & $0.015^{*}$ \\
\hline Lund-Mackay score & $19(13-23)$ & $20(15-24)$ & $20(15-24)$ & $20(16-24)$ & $22(18-24)$ & $<0.001^{*}$ \\
\hline Olfactory score & $1(1-4)$ & $3(1-5)$ & $3(1-5)$ & $1(1-5)$ & $5(4-5)$ & $<0.001^{*}$ \\
\hline Rhinorrhea score & $5(5-6)$ & $5(4-6)$ & $6(5-7)$ & $6(6-7)$ & $6(6-7)$ & $0.0014^{*}$ \\
\hline Nasal obstruction score & $7(6-8)$ & $7(6-8)$ & $7(6-8)$ & $8(7-8)$ & $8(7-8)$ & $<0.001^{*}$ \\
\hline $\begin{array}{l}\text { Facial pain/Headache } \\
\text { score }\end{array}$ & $1(1-4)$ & $1(0-3)$ & $2(1-3)$ & $2(1-4)$ & $3(1-5)$ & $<0.001^{*}$ \\
\hline \multirow[t]{2}{*}{ Asthma (Y/N) } & $4 / 83$ & $1 / 46$ & $13 / 55$ & $5 / 23$ & $47 / 89$ & $<0.001^{*}$ \\
\hline & $(4.6 \%)$ & $(2.1 \%)$ & (19.1\%) & (17.9\%) & (34.6\%) & $<0.001^{*}$ \\
\hline Oral FENO & $23(19-26)$ & $22(20-25)$ & $28(22-39)$ & $24(17-30)$ & $34(28-47)$ & $<0.001^{*}$ \\
\hline Tissue eos\% & $5.56(2.44-11.49)$ & $4.88(1.54-34.58)$ & $40.55(33.31-50.00)$ & $11.20(2.62-28.25)$ & 79.48(68.39-88.24) & $<0.001^{*}$ \\
\hline Tissue neu\% & $3.45(0.0-12.50)$ & $0.0(0.0-0.0)$ & $0.0(0.0-4.84)$ & $48.62(35.87-57.83)$ & $0.0(0.0-0.98)$ & $<0.001^{*}$ \\
\hline Tissue pla \%区 & $42.48 \pm 13.46$ & $17.44 \pm 10.20$ & $17.44 \pm 10.25$ & $13.08 \pm 7.61$ & $6.91 \pm 4.53$ & $<0.001^{*}$ \\
\hline Tissue lym\%区 & $41.57 \pm 14.45$ & $78.15 \pm 10.01$ & $39.67 \pm 15.44$ & $21.04 \pm 12.89$ & $13.87 \pm 9.67$ & 0.498 \\
\hline Peripheral eos $\%$ & $3.2(1.7-4.5)$ & $3.1(1.6-5.6)$ & $6.4(3.7-8.2)$ & $3.8(1.8-6.4)$ & 7.6(5.4-10.4) & 0.979 \\
\hline Peripheral neu\%区 & $52.44 \pm 9.46$ & $53.53 \pm 8.94$ & $51.28 \pm 11.44$ & $53.28 \pm 9.98$ & $50.89 \pm 11.08$ & $<0.001^{*}$ \\
\hline Peripheral lym\%区 & $35.35 \pm 7.28$ & $34.69 \pm 7.24$ & $35.15 \pm 8.43$ & $35.82 \pm 7.81$ & $35.36 \pm 8.08$ & $<0.001^{*}$ \\
\hline Recurrence $(\mathrm{Y} / \mathrm{N})$ & $6 / 81$ & $3 / 44$ & $51 / 17$ & $13 / 15$ & $134 / 2$ & $<0.001^{*}$ \\
\hline
\end{tabular}

Eos, eosinophil. Neu, neutrophil. Pla, plasma cell. Lym, lymphocyte. FENO, fractional exhaled nitric oxide. $\bigotimes$ Data were presented as mean \pm standard deviation. ${ }^{*} \mathrm{P}<0.05$.

\section{Discussion}

CRS remains a common challenging clinical entity due to variable phenotypes with different underlying mechanisms that lead to persistence or recurrence ${ }^{(1)}$. Current guidelines recommend dividing CRS into forms with and without polyps ${ }^{(14)}$. Attempts to further define the classification of CRS have been made by quantifying tissue eosinophilia ${ }^{(10)}$. Moreover, demographic characteristics and medical comorbidities could influence symptom severity, treatment selection and therapeutic response. Thus, better identification of subgroups might permit individualized therapy, with the potential for improvement of patient-tailored care. To date, a study by Nakayama and colleagues ${ }^{(10)}$ has identified 4 clusters of CRS based on eosinophilic inflammation and clinical presentation in Asian patients. Additionally, Soler and colleagues ${ }^{(11)}$ analysed variations in medication usage after defining 5 phenotypes in white patients using cluster analysis. However, neither study has considered prognosis of CRSWNP as a characterization factor. In the present study, we used unsupervised hierarchical cluster analysis to generate 5 clusters with recurrence relevance based on clinical and pathological data from a large cohort of patients with CRSwNP.

Our study demonstrated that cluster 1 and cluster 2 were similar phenotypes with fewer eosinophils in both the local mucosa and peripheral blood. Additionally, tissue neutrophil counts were also low and the two clusters were considered to histologically approximate to "noneosinophilic nonneutrophilic groups" as suggested by lkeda ${ }^{(15)}$. From the clinical viewpoint, patients in these two clusters showed milder symptoms and were less likely to report co-morbid asthma and lower FENO concentrations, 
Table 3. Result of Fisher's linear discrimination analysis of the variables.

\begin{tabular}{|cccccccc} 
Step & $\begin{array}{c}\text { Variable } \\
\text { Included }\end{array}$ & $\begin{array}{c}\text { Tole- } \\
\text { rance }\end{array}$ & $\begin{array}{c}\text { Signi- } \\
\text { ficance } \\
\text { of F to } \\
\text { Re- } \\
\text { move }\end{array}$ & Wilks' $\lambda$ & $\begin{array}{c}\text { Exact F } \\
\text { Value }\end{array}$ & $\begin{array}{c}\text { P } \\
\text { Value }\end{array}$ \\
\hline 1 & Eos\% & 1.000 & $<0.001$ & 0.121 & 657.753 & $<0.001$ \\
\hline 2 & Eos\% & .885 & $<0.001$ & 0.029 & 440.625 & $<0.001$ \\
\hline 3 & Neu\% & .885 & $<0.001$ & & & \\
\hline & Eos\% & .830 & $<0.001$ & 0.015 & 309.451 & $<0.001$ \\
\hline & Neu\% & .857 & $<0.001$ & & & \\
\hline Pla\% & .929 & $<0.001$ & & & \\
\hline Eos\% & .820 & $<0.001$ & 0.014 & 316.758 & $<0.001$ \\
\hline Neu\% & .809 & $<0.001$ & & & \\
\hline Pla\% & .703 & $<0.001$ & & & \\
\hline Lym\% & .686 & $<0.001$ & & & \\
\hline
\end{tabular}

compared to the other three clusters, indicating a milder degree of lower airway inflammation. Importantly, recurrence rates of less than $7 \%$ in these two clusters, was also lower than in the other clusters. Thus, in view of these findings we propose that CRSwNP patients who fall into plasma cell-dominant (plasma cell proportion $\geq 20 \%$ ) and lymphocyte-dominant phenotypes (lymphocyte proportion $\geq 64 \%$ ) have good prognosis and surgery might be the appropriate treatment approach for these individuals.

In comparison, cluster 3 revealed a mixed inflammatory cell pattern. Most cases had tissue eosinophil counts of between $30 \%$ and $54.5 \%$ and lymphocyte counts of less than $64 \%$. This cluster, which also resembled cluster 5 , was characterized by patients with a higher incidence of co-morbid asthma, elevated FENO concentration, higher peripheral eosinophil counts, and more severe olfactory dysfunction, compared to clusters 1, 2 and 4 . This cluster had the second highest recurrence rate of $75 \%$ among all clusters, and as might be expected, the patients in this cluster had a poor prognosis for surgical outcome (prone to recurrence). Importantly, the $85.3 \%$ accuracy of prediction for this cluster using the clinical algorithm was also the lowest across clusters; likely as a consequence of the complicated mix of inflammatory cell types; any of which could present a majority. These findings suggest that the "one size fit all" management approach (medical vs. surgical) used in routine clinical practice is unlikely to be appropriate for patients in this cluster and would need to be tailored accordingly to each individual.

Cluster 4 contained $7.7 \%$ of all patients, who showed the characteristics of neutrophilic CRSwNP. The mean tissue neutrophil count was equal to or greater than $29 \%$ and the mean tissue eosinophil counts less than $30 \%$, according to the algorithm

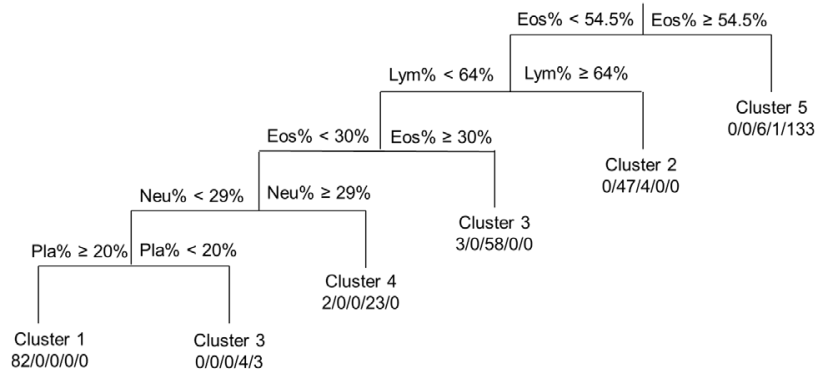

Figure 4. Tree analysis. The subjects were assigned to one of the five clusters using four variables (percentages of mucosal eosinophil, neutrophil, lymphocyte and plasma cell). The number of subjects in each cluster is shown underneath each leaf of the tree (cluster 1/ cluster 2/ cluster 3/ cluster 4/ cluster 5). Eos\%, tissue eosinophil percentage. Neu\%, tissue neutrophil percentage. Pla\%, tissue plasma cell percentage. Lym\%, tissue lymphocyte percentage.

in tree analysis. The vast majority of the patients in this cluster were accurately predictable using above criteria, and nearly half the patients suffered polyp recurrence. The high recurrence rate in this cluster with predominantly neutrophilic inflammation is concordant with findings of other authors, who have reported that patients with neutrophil- or non-eosinophil-dominated nasal polyps have lower response to corticosteroid therapy than patients with eosinophil-dominated nasal polyps ${ }^{(16,17) ;}$ suggesting that a potentially increased resistance to corticosteroid therapy in this cluster may make the patients more prone to severe chronic upper airway disease (SCUAD) ${ }^{(18)}$. The patients in this cluster challenge the current available treatment schemes. SCUAD in this cluster stress the importance of research in the underlying pathophysiological pathway and subsequent disease development.

In contrast, cluster 5 demonstrated the characteristics of eosinophilic CRSwNP; with remarkable tissue and peripheral eosinophilia accompanied by the highest incidence of comorbid asthma (34.6\%), which was similar to white patients in Western countries ${ }^{(11)}$. Moreover, the finding that this cluster had the highest recurrence rate of $98.5 \%$ is in accordance with the findings of previous studies ${ }^{(15,19-21)}$, which have supported the notion that mucosal eosinophilia greatly increased the risk of recurrence in CRSwNP. However, this finding of nearly $100 \%$ recurrence in the present study additionally suggests that a tissue eosinophil count equal to or greater than $54.5 \%$ may be a suitable threshold guide as an indicator for recurrence of nasal polyps after surgery. Conversely,as several studies have also demonstrated that eosinophilic polyps are associated with good responsiveness to steroid therapy $(7,17,22)$, then management with systemic steroid therapy as the mainstay strategy, is likely to be the most appropriate strategy for this group of patients. Our study demonstrated that co-morbid asthma, FENO con- 
Table 4. Accuracy of the discriminant function derived from mucosal eosinophil, mucosal neutrophil, mucosal plasma cells and mucosal lymphocytes.

\begin{tabular}{|c|c|c|c|c|c|c|c|}
\hline \multirow{2}{*}{ Model } & \multirow[t]{2}{*}{ Correct } & \multicolumn{5}{|c|}{ Predicted Group Membership } & \multirow{2}{*}{ Total } \\
\hline & & Cluster 1 & Cluster 2 & Cluster 3 & Cluster 4 & Cluster 5 & \\
\hline \multirow{5}{*}{$\begin{array}{l}\text { Original } \\
\text { Model }^{\mathbf{a}}\end{array}$} & Cluster 1 & 77 & 3 & 5 & 2 & 0 & 87 \\
\hline & Cluster 2 & 1 & 46 & 0 & 0 & 0 & 47 \\
\hline & Cluster 3 & 0 & 4 & 64 & 0 & 0 & 68 \\
\hline & Cluster 4 & 0 & 0 & 0 & 28 & 0 & 28 \\
\hline & Cluster 5 & 0 & 0 & 7 & 0 & 129 & 136 \\
\hline \multirow{5}{*}{$\begin{array}{l}\text { Cross-valida- } \\
\text { ted Model }{ }^{\mathrm{b}}\end{array}$} & Cluster 1 & 76 & 4 & 5 & 2 & 0 & 87 \\
\hline & Cluster 2 & 1 & 46 & 0 & 0 & 0 & 47 \\
\hline & Cluster 3 & 0 & 4 & 64 & 0 & 0 & 68 \\
\hline & Cluster 4 & 0 & 0 & 0 & 28 & 0 & 28 \\
\hline & Cluster 5 & 0 & 0 & 7 & 0 & 129 & 136 \\
\hline
\end{tabular}

The numbers of subjects classified with the original function and with the cross-validation are shown. ${ }^{a} 94.0 \%$ of original grouped cases correctly classified. ${ }^{b}$ Cross validation is done only for those cases in the analysis. In cross validation, each case is classified by the functions derived from all cases other than that case. $93.7 \%$ of cross-validated grouped cases correctly classified.

Table 5. Clinical algorithm performance for predicting clusters a.

\begin{tabular}{|c|c|c|c|c|c|c|}
\hline \multirow{2}{*}{ Cluster } & \multicolumn{5}{|c|}{ Predicted cluster } & \multirow{2}{*}{$\begin{array}{c}\text { Total } \\
\text { (actual) }\end{array}$} \\
\hline & Cluster 1 & Cluster 2 & Cluster 3 & Cluster 4 & Cluster 5 & \\
\hline Cluster 1 & 82 & 0 & 3 & 2 & 0 & 87 \\
\hline Cluster 2 & 0 & 47 & 0 & 0 & 0 & 47 \\
\hline Cluster 3 & 0 & 4 & 58 & 0 & 6 & 68 \\
\hline Cluster 4 & 0 & 0 & 4 & 23 & 1 & 28 \\
\hline Cluster 5 & 0 & 0 & 3 & 0 & 133 & 136 \\
\hline Total & 82 & 51 & 68 & 25 & 140 & 366 \\
\hline
\end{tabular}

a Assignment to clusters based on the clinical algorithm in Figure 4 was compared to the correct cluster as generated through full data analysis. In total, 343/366 cases were correctly assigned (i.e. accuracy of prediction $=93.7 \%$ ).

centration, peripheral eosinophilia, and olfactory dysfunction mirrored tissue eosinophilia across the five clusters. This is in accordance with the well documented evidence that inflammation in the lower respiratory tract affects the upper respiratory tract and vice versa. While one study has demonstrated that CRS was particularly prevalent among people with asthma ${ }^{(2)}$, another demonstrated that when linked to co-morbid asthma, eosinophilic nasal polyps often represented a form of severe eosinophilic airway inflammation and patients experienced a high incidence of recurrence within 5 years ${ }^{(20)}$. The CRS patients with coherent asthma, shown as an important component of SCUAD, have symptoms refractory to standard medical therapy and sinus procedures ${ }^{(18)}$. Our finding for increased incidence of co-morbid asthma and increased levels of FENO, which has been identified as a valid marker of lower airway eosinophilic inflammation, in the present study is in accordance with previous studies, which have shown asthma and FENO to be significantly associated with blood eosinophilia ${ }^{(23)}$, and also reported as a prognostic parameter for recurrence of polyps ${ }^{(20,24,25)}$. Indeed, olfactory impairment has also been shown to be a characteristic symptom of eosinophilic polyps; with statistically significant differences noted for problems with smell between patients with high and low infiltration of eosinophils ${ }^{(26)}$.

Despite the proposal that allergy predisposes to CRS deve- 
lopment, clear and definitive evidences for causality between allergy and CRS, as Bradford-Hill criteria, have not been established so far ${ }^{(27)}$. There exist controversial studies either in favor of or against allergy as a contributing factor for CRS. With regard totreatment outcome, there are conflicting data as to whether systemic atopic status is associated with nasal polyp recurrence. While some studies have shown nasal polyps with allergy to have greater recurrence rate ${ }^{(28,29)}$, others have demonstrated that presence of atopy was not correlated with recurrence ${ }^{(17,20)}$. Herein, our data indicated that systemic atopic status was not statistically significant across the clusters, which was independent of polyp recurrence, and thus suggested that atopy might play a coincidental role, rather than a causative role, in nasal polyp recurrence.

\section{Conclusion}

Our study provided the first proof of concept that informative patterns of tissue inflammatory cells can contribute to objective classification of CRSwNP patients. Five phenotype clusters of CRSwNP, which were distinct for polyp recurrence on the basis of the predominant inflammatory cell type/s in the polyps, can be defined in Chinese patients. These include cluster 1, comprising mostly plasma cell-dominant nasal polyps; cluster 2, comprising mostly lymphocyte-dominant nasal polyps; cluster 3, comprising mixed inflammatory cell type nasal polyps; cluster 4, comprising mostly neutrophil-dominant nasal polyps; and cluster 5, comprising mostly eosinophil-dominant nasal polyps. Although these findings have the potential to aid in the development of individualized treatment strategies for management of CRSWNP patients, these need to be confirmed in further prospective studies and with cohorts comprising patients of different ethnicity.

\section{Acknowledgements}

We thank Dr Hui Chen for her assistance in statistical analysis. This work was supported by grants from the program for Changjiang scholars and innovative research team (IRT13082), the national science fund for the major international joint research program (81420108009), The 12th five year science and technology support program (2014BAI07B04), Beijing Municipal Administration of Hospitals Clinical Medicine Development of Special Funding Support (ZYLX201310), Beijing Municipal Administration of Hospitals' Mission Plan (SML20150203), Beijing Municipal Administration of Hospitals'Youth Programme (QML20150202), national natural science foundation of China (81400444, 81470678), Beijing natural science foundation (7131006), and Beijing health bureau program for high level talents (2011-3-043).

\section{Authorship contribution}

$\mathrm{HL}$ contributed to analyse and interpret the data and prepare the manuscript. YM contributed to acquisition of data. YP provided expertise in pathologic examination. CW, NZ, CB and LZ provided input to study design, subject recruitment, endoscopic sinus surgery, and postoperative follow-up. CW and LZ were responsible for the overall design, data analysis and manuscript preparation.

\section{Conflict of interest}

The authors state that they have no conflict of interest.

\section{References}

1. Akdis CA, Bachert C, Cingi C, et al. Endotypes and phenotypes of chronic rhinosinusitis: a PRACTALL document of the European Academy of Allergy and Clinical Immunology and the American Academy of Allergy, Asthma \& Immunology. J Allergy Clin Immunol 2013; 131:1479-1490.

2. Shi JB, Fu QL, Zhang $H$, et al. Epidemiology of chronic rhinosinusitis: results from a cross-sectional survey in seven Chinese cities. Allergy 2015; 70:533-539.

3. Mygind N. Nasal polyps. In: Mygind N. eds Nasal allergy. Oxford: Blackwell scientific publications, 1978:233-238.

4. Bachert C, Zhang N, Holtappels G, et al Presence of IL-5 protein and IgE antibodies to staphylococcal enterotoxins in nasal polyps is associated with comorbid asthma. J Allergy Clin Immunol 2010; 126:962-968, e961-966.

5. Kountakis SE, Arango P, Bradley D, Wade ZK, Borish L. Molecular and cellular staging for the severity of chronic rhinosinusitis. Laryngoscope 2004; 114:1895-1905.
6. Bassiouni A, Naidoo Y, Wormald PJ. When FESS fails: the inflammatory load hypothesis in refractory chronic rhinosinusitis. Laryngoscope 2012; 122:460-466.

7. Burgel PR, Cardell LO, Ueki IF, Nadel JA. Intranasal steroids decrease eosinophils but not mucin expression in nasal polyps. Eur Respir J 2004; 24:594-600.

8. Kirtsreesakul V, Atchariyasathian V. Nasal polyposis: role of allergy on therapeutic response of eosinophil- and noneosinophildominated inflammation. Am J Rhinol 2006; 20:95-100.

9. Vlaminck S, Vauterin T, Hellings PW, et al. The importance of local eosinophilia in the surgical outcome of chronic rhinosinusitis: a 3-year prospective observational study. Am J Rhinol Allergy 2014; 28:260-264.

10. Nakayama T, Asaka D, Yoshikawa M, et al. Identification of chronic rhinosinusitis phenotypes using cluster analysis. Am J Rhinol Allergy 2012; 26:172-176.

11. Soler ZM, Hyer JM, Ramakrishnan $V$, et al. Identification of chronic rhinosinusitis phenotypes using cluster analysis. Int Forum
Allergy Rhinol 2015; 5:399-407.

12. Lund VJ, Kennedy DW. Quantification for staging sinusitis. The Staging and Therapy Group. Ann Otol Rhinol Laryngol Suppl 1995; 167:17-21.

13. Lund VJ, Mackay IS. Staging in rhinosinusitus. Rhinology 1993; 31:183-184.

14. Fokkens WJ, Lund VJ, Mullol J, et al. EPOS 2012: European position paper on rhinosinusitis and nasal polyps 2012. A summary for otorhinolaryngologists. Rhinology 2012; 50:1-12.

15. Ikeda K, Shiozawa A, Ono N, et al. Subclassification of chronic rhinosinusitis with nasal polyp based on eosinophil and neutrophil. Laryngoscope 2013; 123:E1-9.

16. Wen W, Liu W, Zhang L, et al. Increased neutrophilia in nasal polyps reduces the response to oral corticosteroid therapy. J Allergy Clin Immunol 2012; 129:1522-1528 e1525.

17. Kirtsreesakul V, Atchariyasathian V. Nasal polyposis: role of allergy on therapeutic response of eosinophil- and noneosinophildominated inflammation. Am J Rhinol 2006; 
20:95-100.

18. Prokopakis EP, Vlastos IM, Ferguson BJ, et al. SCUAD and chronic rhinosinusitis. Reinforcing hypothesis driven research in difficult cases. Rhinology 2014;52:3-8.

19. Nakayama T, Yoshikawa M, Asaka D, et al. Mucosal eosinophilia and recurrence of nasal polyps - new classification of chronic rhinosinusitis. Rhinology 2011; 49:392-396.

20. Matsuwaki Y, Ookushi T, Asaka D, et al. Chronic rhinosinusitis: risk factors for the recurrence of chronic rhinosinusitis based on 5-year follow-up after endoscopic sinus surgery. Int Arch Allergy Immunol 2008; 146 Suppl 1:77-81.

21. Stoop $A E$, van der Heijden HA, Biewenga J, van der Baan S. Eosinophils in nasal polyps and nasal mucosa: an immunohistochemical study. J Allergy Clin Immunol 1993; 91:616-622.

22. Wang $C$, Lou $H$, Wang $X$, et al. Effect of budesonide transnasal nebulization in patients with eosinophilic chronic rhinosinusitis with nasal polyps. J Allergy Clin Immunol 2015; 135:922-929 e926.

23. Hastie AT, Moore WC, Li H, et al. Biomarker surrogates do not accurately predict sputum eosinophil and neutrophil percentages in asthmatic subjects. J Allergy Clin Immunol 2013; 132:72-80.

24. Zadeh MH, Banthia V, Anand VK, Huang C. Significance of eosinophilia in chronic rhinosinusitis. Am J Rhinol 2002; 16:313-317.

25. Hu Y, Cao PP, Liang GT, Cui YH, Liu Z. Diagnostic significance of blood eosinophil count in eosinophilic chronic rhinosinusitis with nasal polyps in Chinese adults.
Laryngoscope 2012; 122:498-503.

26. Soler ZM, Sauer DA, Mace J, Smith TL. Relationship between clinical measures and histopathologic findings in chronic rhinosinusitis. Otolaryngol Head Neck Surg 2009; 141:454-461.

27. Georgalas C, Vlastos I, Picavet V, van Drunen C, Garas G, Prokopakis E. Is chronic rhinosinusitis related to allergic rhinitis in adults and children? Applying epidemiological guidelines for causation. Allergy 2014;69:828-33.

28. Dursun E, Korkmaz H, Eryilmaz A, Bayiz $U$ Sertkaya D, Samim E. Clinical predictors of long-term success after endoscopic sinus surgery. Otolaryngol Head Neck Surg 2003; 129:526-531.

29. Pawankar R. Nasal polyposis: an update: editorial review. Curr Opin Allergy Clin Immunol 2003; 3:1-6.
Chengshuo Wang, MD

Department of Otolaryngology Head and Neck Surgery

Beijing TongRen Hospital

No. 1, Dongjiaominxiang

DongchengDistrct

Beijing 100730

China

Tel: (8610) 58268375

E-mail:wangcs830@126.com

Luo Zhang, MD

Department of Otolaryngology Head and Neck Surgery and Department

of Allergy

Beijing TongRen Hospital

Beijing Institute of Otolaryngology

No. 17, HouGouHuTong

DongCheng District

Beijing 100005

China

Tel: (8610) 65141136

Fax: (8610) 85115988

E-mail:dr.luozhang@139.com 